\title{
Behavioral patterns of consumers participating in food safety and social co-governance: consumption choices and environmental improvement
}

\author{
QiuMenghui ${ }^{1}$, and ZhaoZhijing ${ }^{2}$ \\ ${ }^{1}$ Master, Business School, Sichuan Agricultural University, China \\ ${ }^{2}$ Lecturer, Business School, Sichuan Agricultural University, China
}

\begin{abstract}
Consumers are the most direct stakeholders of food safety, and their effective participation is the key to the social Co-governance of food safety. Based on the specific behaviors of Chinese consumers participating directly and indirectly in the social Co-governance of food safety, using the data of 350 consumers' behavior survey and factor analysis and cluster analysis, this paper extracts two kinds of behavior models of Chinese consumers participating in the social Co-governance of food safety, namely, consumption choices model and environment improvement model. It is found that Chinese consumers are inclined to adopt the "environment improvement" model at this stage, which is the inevitable result of the current food safety pressure in China. The government should formulate corresponding policies to better guide consumers to participate in the social Co-governance of food safety.
\end{abstract}

\section{Introduction}

In the context of the growing food safety problem, the government encourages consumers to participate in the "social co-governance" of food safety. From the point of view of existing research, consumer's participation in food safety social co-governance is influenced not only by external environmental factors, but also by consumer' s personal characteristics such as income and ability [1]. Under the comprehensive influence of various factors, consumers often adopt different action strategies. Before the concept of social Co-governance of food safety was put forward, consumers indirectly participated in the social Co-governance of food safety by means of "voting with feet". That is to say, in the process of food consumption, they chose better and safer brand food or labeled food, and refused to consume low-quality, unsafe food, using the role of market supply and demand regulation to encourage food producers to regulate production and operation [2], to achieve indirect governance effect. Recently, more and more direct participation actions have emerged, such as consumer' s supervision of food producers, food safety issues reporting, clue provision, collective rights protection actions and so on.

The essence of indirect participation in the social Co-governance of food safety is to rely on the mechanism of "high quality and high price", and the governance effect is indirect. In addition, due to information asymmetry, income constraints and other factors [3], adverse selection and other market failures are widespread, the mechanism is difficult to fully play its role. With the concept of social Co-governance of food safety put forward, many opinions hold that the way of directly participating in social Co-governance of food safety is more direct, more effective. When consumers and government authorities, expert agencies, media and other subjects participate together, it can form a huge and rigorous social cooperative governance system, directly affects the improvement of the consumption environment, so it is highly expected by all sectors of society.

Indirect participation and direct participation are both important ways for consumers to participate in food safety co-governance, and they complement each other. However, recently, the government regulators put more emphasis on the latter, and many scholars also support the latter in the research. So, how to deal with the relationship between these two ways of participation, advocate consumers to participate directly in food safety and social co-governance has a realistic basis? These questions still need to be answered. Relevant empirical studies are needed to support them.

In order to answer the above questions, this paper systematically combs the specific behavior of consumers participating in food safety social co-governance, and designs the scale of consumer food safety social co-governance behavior. This paper uses field research data, using factor analysis and cluster analysis methods to refine the participation of Chinese consumers in food safety social co-governance behavior patterns, and explore the main ways and participation of Chinese consumers in food safety co-governance at this stage. 


\section{Literature review}

\subsection{Consumer's Indirect participation in the social co-governance of food safety}

Consumers indirectly participate in social Co-governance of food safety mainly through food selection. Specific ways include: Consumers refuse to consume problematic foods and choose branded foods instead of unbranded foods [4]; Choosing certified foods (such as green food, organic food, pollution-free food, etc. ) and traceable foods to replace unlabeled foods [5]; purchasing channels migrate from agricultural markets to large supermarkets; finding alternative food supply system, such as community support agriculture [6]; adopting self-made food and other ways to ensure food safety [7]; choosing to buy foreign food through "Haitao", "Haidai" and other ways. Consumers use the market supply and demand mechanism to influence the production and operation strategies (quality selection) of food producers and operators.

Consumers' food choices may not be the awareness of participating in the social Co-governance of food safety, but only for avoiding food safety risks and ensuring their own safety [8]. However, it is indeed possible to achieve the effect of food safety management through market mechanism. However, there are serious information asymmetry and adverse selection in the food market [9], and Constrained by personal characteristics such as the financial ability of consumers, the regulatory and incentive role of market mechanism is severely inhibited, and the effect of food safety management is not Ideal.

\subsection{Consumer' s direct participation in the social co-governance of food safety}

Consumers' direct participation in the social Co-governance of food safety mainly include: collecting information on food quality and safety, and accumulating relevant experience and skills to reduce food safety risks [10]; Participating in food safety supervision, complaining to the media and relevant departments, providing clues [11]; Consumer collective rights protection, using from the media and other channels to urge the government to strengthen food safety supervision [12]. Consumers, governments and expert institutions jointly manage food safety supervision, and the effect of food safety governance is more effective.To some extent, consumers have to participate in the improvement of the consumer environment to ensure their own safety. Affected by realities, there are some problems such as limited consumer participation, low participation enthusiasm.

From the point of view of existing research, there are two ways for consumers to participate in food safety social co-governance, direct and indirect. Research of the former reveal the governance effect of market supply and demand regulation mechanism on food safety in the specific discussion of consumers' food choice behavior. Research on the latter focuse on the necessity and ways of consumer participation at the theoretical level. The existing research has not systematically combed the specific behavior of consumers participating in food safety social co-governance, and lacks a complete understanding of the overall behavior pattern of consumers participating in food safety social co-governance. At the same time, the main ways and degree of consumers participating in food safety social co-governance in China at this stage are still unclear, and the relevant empirical research has not been reported.

\section{Measurement scales and research methods}

\subsection{Measurement scale}

Firstly, by referring to the existing research and considering the reality, this paper systematically combs out the specific behavior of consumers participating in the social Co-governance of food safety, defines the measurement indicators involved in the scale, and discusses with experts in this field many times to modify the preliminary measurement indicators. The residents around were randomly selected for the prediction test. A total of 88 samples were recovered and 81 valid samples were collected. The recovered sample data were analyzed by exploratory factor analysis. According to factor load matrix, the indexes of cross load or factor load below 0.5 were repeatedly eliminated, and the scale was constantly revised and improved according to the data analysis results. The formal measurement scale containing 14 indicators was shown in Table 1.

\subsection{Research methods}

At present, the research methods of consumer behavior patterns have been relatively mature. This paper mainly refers to Li Baoku et al (2010) [13] research methods to study the patterns of consumer participation in social co-governance behavior. Through cluster analysis of the survey data, we can conclude the behavior pattern of consumers participating in food safety social co-governance. However, there are many measurement items and there may be some correlations among them, exploratory factor analysis is needed to extract the public characteristic factors of behavior patterns, and then cluster analysis of the public characteristic factors, so as to refine and summarize the behavior patterns of consumers participating in the social Co-governance of food safety(See Figure 1). This paper uses SPSS21.0 software to complete data analysis and processing.

\section{Research design and data sources}

Questionnaire items were quantified by Richter Scale 7. The subjects score each item from "totally disagree" to "totally agree" according to 1-7 points. The higher the score, the higher the frequency of the corresponding behavior. Experience shows that this method of fuzzy processing has higher accuracy than direct measurement of the frequency of specific participation behavior. 
Moreover, it can well reflect the specific participation behavior of the subjects under different frequencies of specific participation behavior.

The actual investigation is carried out through paper questionnaire and network survey. Off-line take the way of on-the-spot investigation and on-the-spot recycling, in the large supermarkets, vegetable markets and other places in Chengdu, distribute electronic questionnaires online with the questionnaire star business survey platform. Finally, 380 questionnaires were returned, including 85 electronic questionnaires and 295 paper questionnaires. After eliminating 10 questionnaires with the same score or too short online response time, 350 valid questionnaires were finally recovered, with an effective recovery rate of $91.62 \%$. Most of the respondents were women with middle income or above, living in cities and having children or elderly people in their homes. They have a certain understanding of food safety issues and take certain measures to deal with them. The samples are representative.

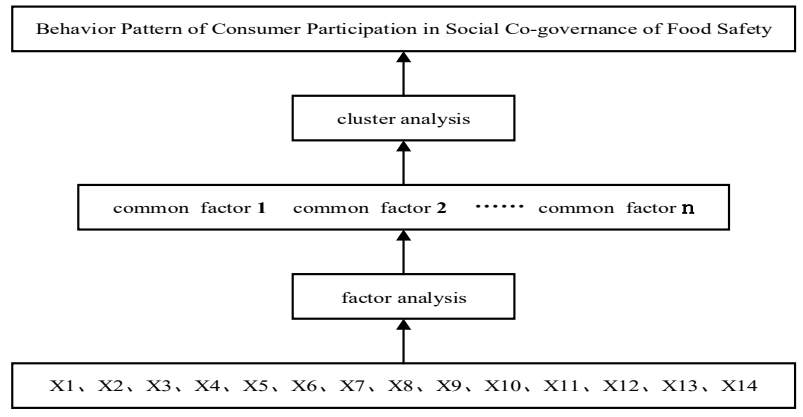

Figure. 1. Schematic diagram of pattern recognition process.

\section{Results analysis}

\subsection{Extraction of common characteristic factor}

Before factor analysis, KMO (Kaiser-Meyer-Olkin) and Bartlett spherical test were used to test the scale. $\mathrm{KMO}=0.747$, Chi-square statistic value of Bartlett's spherical test was 1800.365 , and results were significant $(\mathrm{p}=0.000)$, which was suitable for factor analysis. Four common characteristic factors are extracted and the variance of $65.6 \%$ is cumulatively explained.

As shown in Table 3 . The first common characteristic factor has a relatively large load on X1-X5, called "repurchase factor". The second common characteristic factor has a large load on X12-X14, called "participating regulatory factor". The third common characteristic factor has a large load on X8-X11, called "individualization factor". The fourth common characteristic factor has a large load on X6 and X7, called "import factor".
According to the proportion of the eigenvalues of the four common characteristic factors, we can get the proportion of the public characteristic factors, as shown in formula (1).

$\mathrm{FSSG}=0.440 \mathrm{~F} 1+0.230 \mathrm{~F} 2+0.190 \mathrm{~F} 3+0.141 \mathrm{~F} 4$

Among them, FSSG represents the behavior pattern of consumers participating in the social Co-governance. F1 is the "Subscription Factor", F2 is the "Participation Supervision Factor", F3 is the "Individualization Factor", and F4 is the "Import Factor". The share of "Subscription Factor" is the largest, followed by "Participation in Regulation Factor", and the other two types of public characteristic factors account for less. This shows that in the behavior of consumers participating in the social Co-governance, the proportion of participation in food re-purchase and channel migration is the highest, and the food safety supervision is second, and the purchase of imported food and individualized strategies also account for a certain proportion.

Table 1. Measuring items of consumer food safety social co-governance participation behavior.

\begin{tabular}{|c|c|l|}
\hline & variable & \\
\hline \multirow{4}{*}{$\begin{array}{c}\text { Indirect } \\
\text { participation }\end{array}$} & $\mathrm{X} 1$ & After the usual food brand had safety problems, I reduced or even stopped buying the brand food. \\
\cline { 2 - 3 } & $\mathrm{X} 2$ & After pork safety problems, I reduced or stopped buying pork and bought more beef, mutton and so on. \\
\cline { 2 - 3 } & $\mathrm{X} 3$ & In order to ensure food safety, I usually buy more large-brand foods than non-brand or small-brand ones. \\
\cline { 2 - 3 } & $\mathrm{X} 4$ & In order to ensure food safety, I usually buy more food in supermarkets and other places. \\
\cline { 2 - 3 } & $\mathrm{X} 6$ & To ensure food safety, I often buy foods such as green, organic, pollution-free foods, traceable foods, etc. \\
\cline { 2 - 3 } & $\mathrm{X} 7$ & I am increasingly buying food (milk powder, etc.) through cross-border e-commerce websites. \\
\cline { 2 - 3 } & $\mathrm{X} 8$ & To ensure food safety, I often buy food through relational channels such as friends and relatives \\
\cline { 2 - 3 } & $\mathrm{X} 10$ & to ensure food safety, I usually buy fruits and vegetables from farmers or farms as much as possible. \\
\hline direct & $\mathrm{X} 11$ & I will pay attention to or collect food quality information when I read the news or browse the web. \\
\hline
\end{tabular}




\begin{tabular}{|l|l|l|}
\hline participation & X12 & I usually learn and accumulate tips and experiences on how to remove pesticide residues and select foods. \\
\cline { 2 - 3 } & $\mathrm{X} 13$ & I call 12331, 12315 and other complaints hotlines to report to the regulatory authorities to provide clues. \\
\cline { 2 - 3 } & $\mathrm{X} 14$ & I use more media such as micro-blog, wechat to expose illegal manufacturers \\
\hline
\end{tabular}

Table 2. Variable Variance Scale (\%).

\begin{tabular}{|c|c|c|c|c|c|c|c|c|c|}
\hline \multirow[t]{2}{*}{ component } & \multicolumn{3}{|c|}{ Initial eigenvalue } & \multicolumn{3}{|c|}{ Extract Square Sum Loading } & \multicolumn{3}{|c|}{ Rotating Square Sum Loading } \\
\hline & Total & Variance & Accumulate & Total & Variance & Accumulate & Total & Variance & Accumulate $\%$ \\
\hline 1 & 4.030 & 28.782 & 28.782 & 4.030 & 28.782 & 28.782 & 2.491 & 17.795 & 17.795 \\
\hline 2 & 2.105 & 15.034 & 43.817 & 2.105 & 15.034 & 43.817 & 2.465 & 17.610 & 35.405 \\
\hline 3 & 1.740 & 12.426 & 56.243 & 1.740 & 12.426 & 56.243 & 2.403 & 17.163 & 52.568 \\
\hline 4 & 1.292 & 9.226 & 65.470 & 1.292 & 9.226 & 65.470 & 1.806 & 12.901 & 65.470 \\
\hline 5 & 0.907 & 6.481 & 71.950 & & & & & & \\
\hline 6 & 0.694 & 4.961 & 76.911 & & & & & & \\
\hline 7 & 0.611 & 4.365 & 81.277 & & & & & & \\
\hline 8 & 0.540 & 3.855 & 85.132 & & & & & & \\
\hline 9 & 0.523 & 3.738 & 88.87 & & & & & & \\
\hline 10 & 0.447 & 3.194 & 92.065 & & & & & & \\
\hline 11 & 0.346 & 2.472 & 94.536 & & & & & & \\
\hline 12 & 0.308 & 2.198 & 96.734 & & & & & & \\
\hline 13 & 0.245 & 1.750 & 98.484 & & & & & & \\
\hline 14 & 0.212 & 1.516 & 100 & & & & & & \\
\hline
\end{tabular}

Table 3. Factorial Load Matrix Table after Rotation.

\begin{tabular}{cllllllllllllll}
\hline \multicolumn{2}{c}{ principal component X1 } & $\mathrm{X} 2$ & $\mathrm{X} 3$ & $\mathrm{X} 4$ & $\mathrm{X} 5$ & $\mathrm{X} 6$ & $\mathrm{X} 7$ & $\mathrm{X} 8$ & $\mathrm{X} 9$ & $\mathrm{X} 10$ & $\mathrm{X} 11$ & $\mathrm{X} 12$ & $\mathrm{X} 13$ & $\mathrm{X} 14$ \\
\hline 1 & 0.532 & 0.699 & 0.767 & 0.740 & 0.62 & 0.172 & 0.113 & 0.247 & 0.046 & -0.054 & 0.109 & 0.142 & 0.121 & 0.204 \\
2 & -0.347 & 0.003 & 0.182 & 0.138 & 0.19 & 0.095 & 0.200 & 0.357 & 0.730 & 0.256 & -0.080 & -0.056 & 0.857 & 0.859 \\
3 & 0.297 & 0.179 & -0.006 & 0.015 & 0.07 & 0.066 & 0.111 & 0.562 & 0.308 & 0.693 & 0.805 & 0.847 & -0.049 & -0.001 \\
4 & 0.046 & 0.037 & 0.034 & 0.118 & 0.14 & 0.910 & 0.887 & 0.144 & 0.172 & 0.275 & -0.007 & -0.035 & 0.139 & 0.068 \\
\hline
\end{tabular}

Table 4. Variance Test Table.

\begin{tabular}{lcccccc}
\hline \multicolumn{1}{c}{ Common } & \multicolumn{3}{c}{ mean value } & \multicolumn{3}{c}{ F value t value Sig. (bilateral) } \\
& Cluster 1 (Environmental Improvement) & Cluster 2 (Consumer Choice) & & \\
Subscription Factor & -0.193 & 0.426 & 1.951 & -5.582 & 0 \\
Participation Supervision & 0.206 & -0.457 & 0.839 & 6.028 & 0 \\
Individualization Factor & 0.445 & -0.984 & 0.44 & 16.52 & 0 \\
Import Factor & 0.113 & -0.250 & 1.735 & 3.182 & 0.002 \\
\hline
\end{tabular}

\subsection{Behavior pattern recognition of consumers' participation in social Co-governance of food safety}

This paper conducts rapid cluster analysis on the four common feature factors extracted above. After many calculations with SPSS21.0 software, the results are shown in Table 4. According to the results of variance test, it is basically feasible to aggregate 350 consumers' participation behavior into two categories. There were significant differences between the two types of behavioral patterns in terms of "Subscription Factor", "Participation Supervision Factor", "Individualization Factor" and "Import Factor" $(\mathrm{p}<0.05)$. According to the difference of the mean values of the four common factors between the two types of behavioral patterns, this paper named the two types of behavioral patterns as "consumer choice " and "environmental improvement".

1. Consumer Choice Behavior Pattern. According to Table 4, consumers who adopt such behavioral patterns generally scored higher on the "Subscription Factor". This shows that the consumers who adopt this kind of behavior mode participate in the social Co-governance of food safety to a great extent through food selection behavior, namely, they refuse to consume low-quality food, and encourage food producers to ensure food quality through the regulation mechanism of market supply and demand. Therefore, this paper calls it "consumption choice behavior 
pattern". Of the 350 consumers surveyed, 109 (30.14\%) consumers' food safety social co-governance participation behavior pattern belong to this type(see Table 5).

2.Environmental Improvement Behavior Pattern. According to Table 4, consumers who adopt such behavioral patterns scored the highest on the "individualization factor", followed by the "participation regulatory factor" and the "import factor" scored a certain proportion. This shows that consumers who adopt this kind of behavior pattern choose to collect food quality information, change food safety supply channels and other individualized strategies to a great extent. Many consumers choose to participate directly in assisting food safety supervision. Some consumers choose to buy foreign food directly. Consumers are willing to spend extra time, energy and financial resources to exert pressure on food producers and operators, and strive to improve the food consumption environment quickly and effectively. Therefore, this paper calls it "environmental improvement behavior pattern", which is based on individualized behavior strategy. There are relatively few acts involved in assisting food safety supervision, and the behavior of choosing to buy foreign food is not common. $241 \quad(68.86 \%)$ consumers' social co-governance participation behavior pattern belong to this type (see Table 5).

Table 5. Final results of fast cluster.

\begin{tabular}{cccc}
\hline \multicolumn{2}{c}{ Number of cases in each cluster } & Proportion \\
\hline cluster & 1 & 241 & $68.86 \%$ \\
& 2 & 109 & $31.14 \%$ \\
effective & & 350 & $100 \%$ \\
Defect & 0 & 0 \\
\hline
\end{tabular}

\section{Conclusions and policy implications}

\subsection{Conclusions}

Based on the specific behavior of Chinese consumers directly and indirectly participating in the social Co-governance of food safety, this paper use the data of 350 consumers'behavior survey, extracting two kinds of behavior models of Chinese consumers participating in the social Co-governance of food safety, namely, consumption choice and environment improvement pattern. The mode of "consumption choice" has evolved from the early dominant position to the secondary position at the present stage. At present, Chinese consumers prefer to adopt the pattern of "environment improvement", in which the individualized strategy is the main participation behavior of adopting this pattern, while the actual participation of assisting food safety supervision is low.

\subsection{Policy enlightenment}

Policy implications of this study:

Firstly, "consumer choice" and "environment improvement" are both important behavioral patterns of Chinese consumers participating in food safety co-governance. the government should focus on solving the problem of market failure caused by information asymmetry in the "consumer choice" model, further improve the mechanism of food safety information disclosure, help the public to timely access to food quality and safety information, and improve the governance effect of the "consumer choice" model.

Secondly, At present, the participation of consumers in individualized strategies is more, while the actual participation of food safety supervision assistance is lower. It not only shows that consumers prefer to express their dissatisfaction through individual concealment behaviors, but also shows that there are too many obstacles for consumers to participate in food safety co-governance directly. The government should try to improve consumer' $\mathrm{s}$ participation from the Perspective of improving existing policies.

\section{References}

1. Z.Q. Yu, Hebei University of Economics and Trade, (2018)

2. L. Unnevehr, V. Hoffmann, J. Integr. Agric, 11, 14 (2015)

3. J. Ma, F. Qin, Chinese Rural Economy, 05 (2009)

4. M. Niewczas, Int. J. Consum. Stud, 3, 38 (2014)

5. D. Menozzi, R. Halawany-Darson, C. Mora, Food Control, Sp. Iss. SI. 49 (2015)

6. L. Zhang, G. Qi, Can. J. Dev. Stud, 1, 40 (2019)

7. M. Jin, S.Y. Qian, Shanxi University of Finance \& Economics, 01, 40 (2018)

8. S.W. Quan, Y.C. Zeng, Chinese Population, Resources and Environment, 04 (2014)

9. P. Nelson, J. Polit. Econ, 2, 78 (1970)

10. De Jonge J. Amonitor for consumer confidence in the safety of food, 2008.

11. K. Chen, X.X. Wang, H. Song, J. Integr. Agric, 11, 14 (2015)

12. K. Xie, J.T. Lai, J.H. Xiao, Economic research, 04 (2016)

13. C.X. Zhai, B.K. Li, Y. Liu, Management Review, 01, 22 (2010) 